\title{
Portal Vein Thrombosis: An Unexpected Finding in a 28-Year-Old Male With Abdominal Pain
}

\author{
Jason L. Ferguson, DO, and Duane R. Hennion, MD
}

Background: Abdominal pain is a common primary care complaint. Portal vein thrombosis (PVT) is a rare cause of abdominal pain, typically associated with cirrhosis or thrombophilia. The following describes the presentation of PVT in a young male, the search for risk factors and underlying etiology, and the debate of anticoagulation therapy.

Case: A 28-year-old male presented with periumbilical pain, post-prandial nausea, and sporadic hematemesis for 3 weeks. The diagnosis was confirmed with a triphasic liver computerized tomography after obtaining an abnormal right upper quadrant ultrasound. This unexpected finding prompted investigation for intrinsic hepatic disease and potential hypercoagulable disorders. Laboratory analysis revealed a heterozygous genotype for the prothrombin 20210G/A mutation, an identified risk factor for venous thrombosis.

Discussion: Recommendations concerning anticoagulation for PVT in the absence of cirrhosis are not clearly defined. Current literature describes the following factors as indications for anticoagulation: acute thrombus, lack of cavernous transformation, absence of esophageal varices, and mesenteric venous thrombosis. This patient had clinical indications both for and against anticoagulation. Weighing this individual's clinical circumstances, we concluded the risk of thrombus in the setting of a hypercoagulable disorder outweighed the risk of variceal bleeding. A minimum of 6 months of anticoagulation was initiated.

Conclusion: PVT is an uncommon cause of abdominal pain, and the absence of hepatic disease should raise the index of suspicion for an underlying thrombophilia. Specific recommendations for anticoagulation are not well defined, demonstrating the importance of weighing the individual risks and benefits in treatment with anticoagulation for young persons with thrombophilia. ( $\mathrm{J}$ Am Board Fam Med 2008;21:237-243.)

Abdominal pain has been shown in the general population to make up between $4.4 \%$ and $7.5 \%$ of primary care and emergency department visits. ${ }^{1-3}$ Although the spectrum of disease processes producing abdominal pain is vast, portal vein thrombosis (PVT) is not commonly considered in the differential diagnosis among the average, otherwise healthy, adult population. Although PVT has been well documented since 1868 , the entity is usually associated with cirrhosis, liver transplantation, malignancies, inflammatory disorders, or hypercoagu-

This article was externally peer reviewed.

Submitted 26 June 2007; revised 25 December 2007; accepted 7 January 2008.

From the Department of Family Medicine, Tripler Army Medical Center, Honolulu, HI.

Funding: none.

Conflict of interest: none declared.

Corresponding author: Jason L. Ferguson, DO, Department of Family Medicine, Tripler Army Medical Center, Honolulu, HI 96859 (E-mail: jjferg23@yahoo.com). lable states. The prevalence of idiopathic PVT is not well known but experts agree it is rare. ${ }^{12,16}$ The following case report describes the clinical presentation of a young adult male with abdominal pain and the subsequent discovery of PVT, the etiology of which can probably be attributed to a heterozygous prothrombin gene mutation. In addition to the statistical contrast between this common presenting complaint and rare subsequent diagnoses, this case is distinct because recommendations for anticoagulation are vaguely defined for individuals with the heterozygous prothrombin gene and venous thromboses.

\section{Case Report}

A 28-year-old white male presented with a chief complaint of a 3-week history of mid-epigastric pain described as a "tight band" surrounding his abdomen. This 3-inch horizontal, band-like distri- 
bution included the umbilicus and radiated to the patient's back bilaterally. The abdominal pain was associated with meals, consisting of a significant increase in pain approximately 10 to 15 minutes after eating and intermittent post-prandial nausea and occasional vomiting. The patient reported a single episode of hematemesis and 2 melena stools approximately 1 week before presentation to the clinic. He denied hematochezia, changes in stool consistency or frequency, weight loss, night sweats, or easy bruising or bleeding. There was no history of recent travel, unusual environmental exposures, or any trauma to the abdomen. The patient noted 1 month of increasing fatigue and a 10-pound weight loss, which he had attributed to altering his diet to find meals that did not exacerbate his pain or nausea. His medical history was significant for gastroesophageal reflux disease diagnosed 4 years earlier. He has smoked one-half of a pack of cigarettes per day and has drank 2 to 3 glasses of beer on the weekends for the last 3 years. The patient denied having any previous surgeries nor was he taking any medications at the time of presentation. His family history was noncontributory, specifically for bowel disease or bowel cancer, and negative for any history of bleeding or clotting disorders.

On physical examination, the patient was noted to be afebrile with a normal blood pressure and a heart rate of 56, which was thought to be physiologic per the patient's aerobic fitness level. Specific examination findings were sparse spider angiomas involving the upper chest and neck and some mild tenderness to palpation over the periumbilical and epigastric region. The patient did not have an appreciably enlarged liver or spleen on palpation. He did not demonstrate signs of icteric or pale sclera nor did he have jaundiced appearance or pale palmar creases. He did not have signs of asterixis. A rectal examination was negative for gross blood and was also negative for occult blood. Laboratory studies in general showed sings of hypersplenism with a mildly decreased white blood cell count and thrombocytopenia and also demonstrated a mild transaminitis. His blood cell lines did not suggest a severe blood loss given his history, nor did they suggest an infectious, inflammatory, or malignant process in regards to his chief complaint. See Table 1 for initial laboratory results.

A right upper quadrant ultrasound was performed to evaluate for cholelithiasis because the history of post-prandial pain was concerning for
Table 1. Initial Laboratory Results

\begin{tabular}{lcc}
\hline Laboratory Exam & Result & Reference Range \\
\hline White blood cell count & $3,200 \mathrm{cells} / \mathrm{mm}^{3}$ & 3.9 to 10.6 \\
Hemoglobin & $14.5 \mathrm{~g} / \mathrm{dL}$ & 13.3 to 17.7 \\
Hematocrit & $43.2 \%$ & 40.0 to 53.1 \\
Platelets & $99,000 / \mathrm{mm}^{3}$ & 150 to 440 \\
AST & $35 \mathrm{IU} / \mathrm{L}$ & 0 to 37 \\
ALT & $51 \mathrm{IU} / \mathrm{L}$ & 0 to 51 \\
GGT & $38 \mathrm{IU} / \mathrm{L}$ & 11 to 51 \\
Alkaline phosphatase & $127 \mathrm{IU} / \mathrm{L}$ & 38 to 126 \\
Total bilirubin & $0.8 \mathrm{mg} / \mathrm{dL}$ & 0.2 to 1.0 \\
Direct bilirubin & $0.2 \mathrm{mg} / \mathrm{dL}$ & 0 to 0.3 \\
Amylase & $47 \mathrm{IU} / \mathrm{L}$ & 30 to 110 \\
Lipase & $29 \mathrm{IU} / \mathrm{L}$ & 0 to 190 \\
Blood urea nitrogen & $10 \mathrm{mg} / \mathrm{dL}$ & 6 to 20 \\
Creatine - serum & $0.7 \mathrm{mg} / \mathrm{dL}$ & 0.8 to 1.4 \\
Prothrombin time & $13.9 \mathrm{sec}$ & 11.7 to 14.2 \\
INR & 1.1 & 1.0 \\
Partial thromboplastin time & $30 \mathrm{sec}$ & 24 to 36 \\
\hline
\end{tabular}

AST, aspartate transaminase; ALT, alanine aminotransferase; GGT, gamma glutamyltransferase; INR, international normalized ratio.

biliary colic. The ultrasound demonstrated a normal-appearing liver and gallbladder but was significant for an enlarged spleen with an apparent small portal vein and several serpiginous vessels, suggestive of PVT with cavernous transformation. This finding was further confirmed with a triphase liver computerized tomography (CT) scan which showed a decrease of opacification in the main portal vein with several collateral vessels in the splenic hilum as well as the porta hepatis. The scan further described thrombosis into the superior mesenteric vein as well (see Figure 1).

In light of the finding of PVT with the prior history of hematemesis and melena, the patient underwent esophagogastroduodenoscopy, which revealed Grade II-III nonbleeding esophageal varices in the distal esophagus. Variceal band ligation (VBL) was performed for suspected recurrent bleeding and as secondary prophylaxis. With an unknown etiology for PVT, additional laboratory serum studies were performed to search for hypercoagulable disorders as well as evidence of intrinsic hepatic disease. The ensuing laboratory studies were normal, with the exception that the patient was determined to be heterozygous for the prothrombin 20210G/A gene mutation (see Table 2). This genetic mutation has been shown to be a risk 


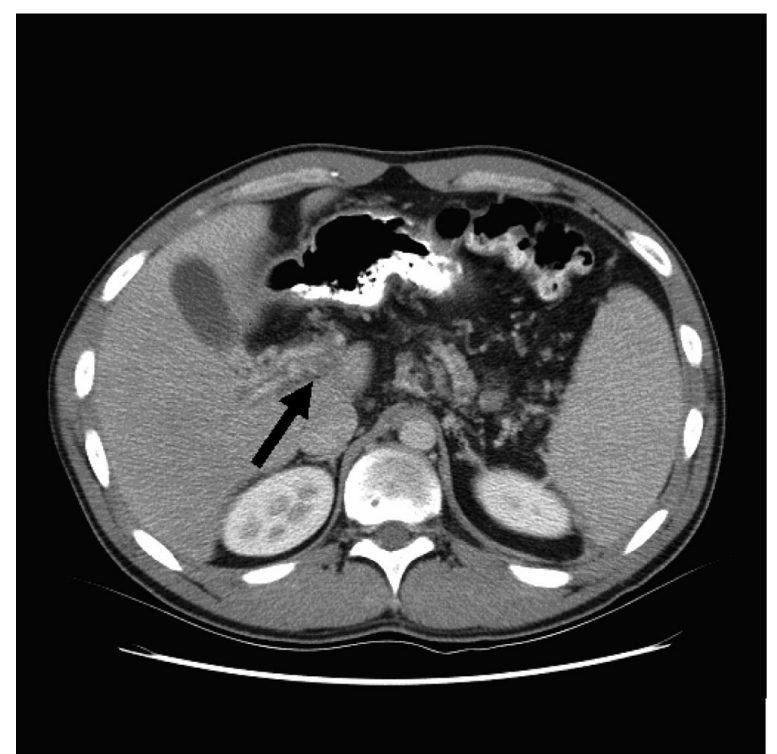

Figure 1. Selected computed tomography image with arrow indicating thrombosis within the portal vein and evidence of cavernous formation.

factor for thrombosis, albeit current studies show discrepancies in the degree or significance of risk.

Anticoagulation treatment options raised significant debate regarding the benefits of preventing further thromboses versus the risk of subsequent bleeding. After literature review and extensive consultation with gastroenterology, hematology, and the patient, anticoagulation therapy was initiated. The patient was started on low-molecular-weight heparin as an inpatient and converted to oral anticoagulation therapy using warfarin before discharge home with a target international normalized ratio (INR) of 2 to 3 , with the goal of preventing further clot formation in the portal and mesenteric venous system. After 2 weeks, the severity and frequency of the patient's post-prandial pain and nausea had decreased significantly. He was completely asymptomatic by 1-month postdischarge. A repeat CT of the abdomen is currently pending to evaluate resolution of the thrombus. The patient will remain on warfarin therapy with an INR goal of 2.0 to 2.5 for 6 to 12 months.

\section{Discussion}

PVT as the etiology of abdominal pain in an otherwise healthy young adult is an uncommon occurrence and raises several questions. What are the etiologies for and prevalence rates of PVT, especially in a noncirrhotic patient? What is the significance of the prothrombin 20210G/A mutation and the impact on relative risk of thrombosis? What are the treatment options for a patient with noncirrhotic PVT and the prothrombin gene mutation as the leading etiology?

\section{Noncirrbotic PVT}

Thrombus formation in the portal venous system was first described in $1868^{4}$ and has been well documented since. It is most commonly associated with cirrhosis, with PVT ranging from $11 \%$ to $6 \%$ in known cirrhotic patients. ${ }^{11,12,14}$ Outside the realm of cirrhosis, PVT is thought to be so rare in the general population that its prevalence has not been defined. Known etiologies can be divided into 2 broad categories to include thrombophilic disorders and thromboses thought to be caused from local factors (see Table 3).

It has been estimated that in noncirrhotic PVT patients, thrombophilic states account for approxi-

Table 2. Hypercoagulable Laboratory Results

\begin{tabular}{|c|c|c|}
\hline Study & Result & Interpretation \\
\hline Factor V Leiden mutation & Genotype: R/R & Normal genotype \\
\hline Prothrombin mutation & Genotype: G/A & Heterozygous positive \\
\hline Antithrombin III & $83 \%$ Activity seen & Normal activity \\
\hline Protein C Deficiency & $87 \%$ Activity seen & Normal activity \\
\hline Protein S Deficiency & 107\% Activity & Normal activity \\
\hline Homocysteine & $7.3 \mu \mathrm{mol} / \mathrm{dL}$ normal value & Normal value \\
\hline \multirow[t]{3}{*}{ Anticardiolipin antibody } & IgA: 1.9 & Normal limits \\
\hline & IgG: 2.8 & \\
\hline & IgM: 1.8 & \\
\hline $\begin{array}{l}\text { Lupus anticoagulant antibody screening through } \\
\text { Russell's viper venom assay }\end{array}$ & 35 seconds & Normal \\
\hline
\end{tabular}




\begin{tabular}{lllll}
\hline \multicolumn{2}{c}{ Thrombophilic Disorders } & & \multicolumn{2}{c}{ Local Factors } \\
\cline { 1 - 2 } Inherited Disorders & \multicolumn{1}{c}{ Acquired Disorders } & & \multicolumn{1}{c}{ Inflammatory } & Related to Surgery \\
Factor V Leiden mutation & Myeloproliferative disorders & & Cirrhosis & Post liver transplant \\
Prothrombin mutation & Malignancy & & Sepsis & Splenectomy \\
Antithrombin III & Antiphospholipid syndrome & & Pancreatitis/cholecystitis & Colectomy \\
Protein C deficiency & Anticardiolipin antibody & & Diverticulitis & Umbilical vein catheterization \\
Protein S deficiency & Paroxysmal nocturnal hemoglobinuria & Appendicitis & Portacaval shunting \\
& Hyperhomocysteinemia & & Peptic ulcer disease & \\
& Oral contraception pills & & Inflammatory bowel disease & \\
& Pregnancy/postpartum & & Blunt abdominal trauma & \\
\hline
\end{tabular}

mately $40 \%$ to $60 \%$ of PVT cases, and local factors are thought to be the causative factor in $10 \%$ to $50 \%{ }^{8,14}$ Since the 1980 s and the discovery of several inherited thrombophilic factors such as factor V Leiden and the prothrombin G20210 gene mutations, several authors have reported a decreasing proportion of idiopathic PVT ranging between $8 \%$ and $15 \%{ }^{8,12}$ Specifically regarding PVT, the prothrombin mutation has been shown to be the only explainable cause of PVT in a number of cases that would otherwise be named idiopathic. Retrospectively, Chamouard et $\mathrm{al}^{31}$ found the prothrombin mutation to be present in $40 \%$ of patients presenting with a prior diagnosis of idiopathic PVT.

Clinically, PVT has been described in 2 general presentations: acute and chronic. In practice however, these 2 categories are difficult to distinguish with common symptoms and are often delineated with imaging studies. Clinical symptoms of acute thrombosis include abdominal pain, often localized to the epigastrium or umbilical region, with associated anorexia and nausea/vomiting. These symptoms are especially common when the thrombus involves the superior mesenteric vein and produces bowel ischemia via venous congestion. ${ }^{8,18}$ Chronic PVT has similar subjective complaints but are frequently accompanied by problems related to portal hypertension and hypersplenism. These symptoms and findings include gastrointestinal bleeding, splenomegaly, thrombocytopenia, anemia, and leukopenia. From a pathophysiology perspective, a distinguishing feature of chronic PVT is the presence of a dense complex of tortuous collateral veins bypassing the thrombosed region called a cavernoma. ${ }^{8,16}$ As in this case, PVT is often diagnosed with Doppler ultrasound imaging which shows absent or decreased flow across the portal vein. However, CT may be more useful in demonstrating the develop- ment of a cavernoma and surrounding collateral vessels.

Important consequences of chronic PVT have been primarily related with bowel ischemia and bleeding from esophageal varices, with the latter thought to manifest in $30 \%$ of PVT cases. ${ }^{12}$ Varices with established PVT are often large, which is an independent risk factor for rebleeding risk. ${ }^{15}$ However, it has been objectively documented that large varices secondary to PVT have lower frequency of bleeding when treated similarly compared with patients with similar grade varices secondary to liver cirrhosis, $0.25 \%$ and $20 \%$ to $30 \%$ of rebleeding, respectively. ${ }^{15}$ These differences are thought to be primarily because of the overall morbidity and complications of an intrinsic liver disease versus an isolated and often reversible prothrombotic state. Treatment for varices secondary to PVT is similar to techniques for varices from other portal hypertension processes and employs endoscopy eradication with VBL, beta-blockers, and nitrates. One study suggests that these techniques are low risk and highly successful, demonstrating a 5 -year survival rate of $95 \%$ and no mortality related to recurrent bleeding in extrahepatic portal vein obstruction. ${ }^{19}$ Six months posttreatment, the presented patient remained asymptomatic and had no recurrence of hematemesis or melena after VBL of his Grade II-III varices. Beta-blockers were not prescribed because of his low resting heart rate.

\section{Prothrombin G20210A Mutation}

There is an ongoing controversy in the literature regarding the underlying significance of the prothrombin mutation. The literature has predominantly supported the idea that presence of the prothrombin mutation causes an increased risk for thrombosis by itself, with relative risks for venous 
thrombosis of 2- to 4-fold. ${ }^{23,26}$ However, Vaya et $\mathrm{al}^{29}$ reported a case study with a homozygous carrier of the prothrombin gene with severe venous thrombosis, which contrasts several previous case studies of homozygous carriers of the prothrombin gene mutation with no prior history of thromboses. There are currently only small, retrospective, cohort studies available from which to extrapolate data.

\section{Treatment for PVT with the Prothrombin Mutation}

Treatment of PVT can be approached with several different methods, ranging from thrombectomy and thrombolytic therapy, to transjugular intrahepatic portosystemic shunting (TIPSS), or prolonged anticoagulation. This may in part be a result of the lack of randomized, controlled studies of any method of treatment for PVT, relying mainly on small retrospective studies and case reports. When narrowing the literature search to PVT in the setting of a single inherited prothrombotic risk factor such as the prothrombin gene mutation, recommendations for treatment are vague and are driven mainly by expert opinion. Multiple controversies exist in regards to the appropriate evidence-based treatment of PVT.

Anticoagulation has proven beneficial in both prevention of further extension of a thrombus, as well as aiding recanalization of the thrombus. ${ }^{8-10,12-15}$ A significant controversy regarding anticoagulation resides in whether or not the thrombus is acute or chronic. Chronic PVT is often associated with portal hypertension and esophageal varices, which are a significant risk factor for bleeding with anticoagulation.

The effectiveness of anticoagulation in patients with evidence of acute PVT has been reported in several small case studies. Sheen et $\mathrm{al}^{9}$ demonstrated that $78 \%$ of noncirrhotic patients had resolution of their thrombus per CT reassessment after 3 months of oral anticoagulation with an INR range of 2 to 4 . Condat et $\mathrm{al}^{10}$ report in a retrospective study of acute PVT that recanalization occurred in 25 of 27 patients given anticoagulation and 0 of 2 patients without anticoagulation therapy. These reports support the argument for anticoagulation as an effective and noninvasive approach to treatment but are lacking in several aspects to include an exact duration of treatment. These studies are also small and nonprospective without clear recommendations for INR ranges and do not ad- dress the need for possible future prophylactic anticoagulation.

Despite the supportive data in the acute PVT setting, there is considerable concern regarding anticoagulation risks in well-established or chronic PVT, primarily based on the fear of gastrointestinal bleeding in a portal hypertensive state. In 2001, Condat et $\mathrm{al}^{15}$ described rates of bleeding in a retrospective cohort study of spanning 15 years and 136 cases of nonmalignant and noncirrhotic PVT. Eighty-four of the patients received anticoagulation therapy and the remainder did not. Eightyfour bleeding episodes were reported in 42 patients for an incidence rate of 12.5 per 100 patient years; however, there was no increase in risk or severity of bleeding with anticoagulation when comparing those anticoagulated versus those who were not. It was further concluded that the incidence of new thrombus formation was reduced and that large esophageal varices are an independent risk factor for bleeding. Although the evidence is not definitive, these data support the idea that the risk-benefit ratio favors anticoagulation in chronic noncirrhotic PVT. Combined with additional expert opinion resources, recommendations are that anticoagulation should begin after large esophageal varices are treated similarly to other portal hypertensive states with variceal ligation banding or sclerotherapy to further aid in reduction of acute bleeding. ${ }^{12}$ Although the above studies have varying durations of anticoagulation, the above data seem to suggest a minimum of 6 months of oral anticoagulation with repeat endoscopy and CT imaging to evaluate for signs of thrombus resolution.

"Is prophylactic anticoagulation warranted in persons with PVT whose etiology is probably secondary to the prothrombin mutation?" remains a difficult question. Current literature provides conflicting recommendations to this question, and most of the expert opinion specifically addresses more common genetic mutations such as factor $\mathrm{V}$ Leiden deficiency or in combination with the prothrombin mutation. A conservative statement is often found stating that prolonged or lifetime anticoagulation should be considered with persons with a prothrombotic etiology. ${ }^{7,13,15}$ Contrastingly, 2 studies in particular suggest with prospective data that risk of recurrent thromboses in individuals with the G20210A prothrombin mutation does not differ from individuals without genetic thrombophilia. ${ }^{33,34}$ These studies recommend that both cat- 
egories of people should be treated following the current recommendations for deep venous thromboses in regards to duration of therapy. Interestingly, De Stefano et $\mathrm{al}^{32}$ have also concluded in a separate writing that the risk of recurrent deep venous thrombosis is increased in carriers of both factor V Leiden and the G20210A prothrombin mutations after a first episode of thrombosis and thus are candidates for lifelong anticoagulation. This thought would also concur with the recent recommendations of the American College of Chest Physicians for the consideration of indefinite anticoagulation for any first time, idiopathic venous thrombosis because many idiopathic events may have an underlying attributable etiology such as the prothrombin gene mutation. ${ }^{38}$

\section{Conclusion}

This case report serves as a reminder that a common presenting symptom such as abdominal pain in a young, healthy adult can be a manifestation of a rare diagnosis such as PVT. Literature supports that PVT outside the realm of liver cirrhosis is typically because of a hypercoagulable state, which warrants a methodic search for the specific thrombophilic etiology. The prothrombin gene mutation is a relatively new discovery that has been established in the literature as a risk factor for venous thrombosis, but the rate of recurrence for thrombosis and possible need for prophylactic anticoagulation are still under debate. Current literature regarding the management of the prothrombin gene mutation and PVT is based primarily on small retrospective analyses.

\section{References}

1. Graham R, Roberts R, Ostergaard D, Kahn N Jr, Pugno P, Green L. Family practice in the United States: a status report. JAMA 2002;288:1097-101.

2. Black P. Chief complaints in a free walk-in clinic. Public Health Rep 1977;92:150-3.

3. McCaig B. Statistics on emergency and trauma care utilization. In: Future of emergency care: hospitalbased emergency care at the breaking point. Institute of Medicine. Washington, DC: The National Academies Press; 2007. p. 345-52.

4. Balford GW, Stewart TG. Case of enlarged spleen complicated with ascites, both depending on varicose dilation and throbosis of the portal vein. Edinb Med J 1869;14:589-98.

5. Primignani M, Martinelli I, Bucciarelli P, et al. Risk factors for thrombophilia in extrahepatic portal vein obstruction. Hepatology 2005;41:603-8.

6. Walker A. Portal vein thrombosis: what is the role of genetics?. Eur J Gastroenterol Hepatol 2005;17:705-7.

7. Valla DC. Portal vein thrombosis and prothrombic disorders. J Gastroenterol Hepatol 1999;14:1051-2.

8. Valla DC, Condat B. Portal vein thrombosis in adult: pathophysiology, pathogenesis and management. J Hepatol 2000;32:865-71.

9. Sheen CL, Lamparelli H, Milne A, Green I, Ramage JK. Clinical features, diagnosis and outcome of acute portal vein thrombosis. Q J Med. 2000;93:531-4.

10. Condat B, Pessione F, Denninger MH, Hillaire S, Valla DC. Recent portal or mesenteric venous thrombosis: increased recognition and frequent recanalization on anticoagulant therapy. Hepatology 2000;32:466-70.

11. Okuda K, Ohnishi K, Kimura K, et al. Incidence of portal vein thrombosis in liver cirrhosis. An angiographic study in 708 patients. Gastroenterology 1985;89:279-86.

12. Webster GJM, Burroughs AK, Riordan SM. Review article: portal vein thrombosis - new insights into aetiology and management. Aliment Pharmacol Ther 2005;21:1-9.

13. Hegenbarth K, Fickert P, Aschauer M, Horina JH, Stauber RE, Trauner M. Successful management of acute portal vein thrombosis by low molecular weight heparin and oral anticoagulation. Am J Gastroenterol 2002;97:1567-8.

14. Kocher G, Himmelmann A. Portal vein thrombosis (PVT): a study of 20 non-cirrhotic cases. Swiss Med Wkly 2005;135:372-6.

15. Condat B, Pessione F, Hillaire S, et al. Current outcome of portal vein thrombosis in adults: risk and benefit of anticoagulant therapy. Gastroenterology 2001;120:490-7.

16. Sobhonslidsuk A, Reddy R. Portal vein thrombosis: a concise review. Am J Gastroenterol 2002;97:535-41.

17. Denninger MH, Chait Y, Casadevall N, et al. Cause of portal or hepatic venous thrombosis in adults: the role of multiple concurrent factors. Hepatology 2000;31:587-91.

18. Choudhary A, Grayer D, Nelson A, Roberts I. Mesenteric venous thrombosis a diagnosis not to be missed. J Clin Gastroenterol 2000;31:179-82.

19. Vleggaar FP, van Buuren HR, Schalm SW. Endoscopic sclerotherapy for bleeding esophagogastric varices secondary to extrahepatic portal venous obstruction in an adult population. Eur J Gastroenterol Heptaol 1998;10:81-5.

20. Zivelin A, Rosenberg N, Faier S, et al. A single genetic origin for the common prothrombotic G20210A polymorphism in the prothrombin gene. Blood 1998;92:1119-24.

21. Tateishi A, Mitsui H, Oki T, et al. Extensive mesenteric vein and portal vein thrombosis successfully 
treated by thrombolysis and anticoagulation. J Gastroenterol Hepatol 2001;16:1429-33.

22. Perry S, Ortel T. Clinical and laboratory evaluation of thrombophilia. Clinics Chest Med 2003;24:15370.

23. Feero G. Genetic thrombophilia. Prim Care Clin Office Pract 2004;31:685-709.

24. Bick R. Prothrombin G20210A mutation, antithrombin, heparin cofactor II, protein C, and protein S defects. Hematol Oncol Clin N Am 2003;17: 9-36.

25. Poort S, Rosendaal F, Reitsma P, Bertina R. A common genetic variation in the 3 '-untranslated region of the prothrombin gene is associated with elevated plasma prothrombin levels and an increase in venous thrombosis. Blood 1996;88:3698-703.

26. Brown K, Luddington R, Williamson D, Baker P, Baglin T. Risk of venous thromboembolism associated with a $G$ to $A$ transition at position 20210 in the 3' untranslated region of the prothrombin gene. Br J Haematol 1997;98:907-9.

27. El Hajj I, Salem Z, Birjawi G, Taher A, Soweid A. Heterozygous prothrombin 202010G/A mutation, associated with hyperhomocysteinemia, and homozygous methylenetetrahydrofolate reductase $677 \mathrm{C} / \mathrm{T}$ mutation, in a patient with portal mesenteric venous thrombosis. Hematol J 2004;5:540-2.

28. Rosendaal FR, Doggen CMJ, Zivelin A, Arruda VR, Aiach M, Siskovick DS Geographic distribution of the 20210G to A prothrombin variant. Thromb Haemost 1998;79:706-8.

29. Vaya A, Garcia M, Mira Y, et al. Homozygous 20210G/A prothrombin gene mutation associated with bilateral iliac vein thrombosis: a case report. Thromb Res 2001;104:293-6.

30. Ranguelov R, Rosenthal N, Bromley C, Vasef M.
Detection of factor $\mathrm{V}$ Leiden and prothrombin gene mutations in patients who dies with thrombotic events. Arch Pathol Lab Med 2002;126:1193-6.

31. Chamouard P, Pencreach E, Maloisel F, et al. Frequent factor II G20210A mutation in idiopathic portal vein thrombosis. Gastroenterology 1999; $116: 144-8$

32. De Stefano V, Martinelli I, Mannucci P, et al. The risk of recurrent deep venous thrombosis among heterozygous carriers of both factor $\mathrm{V}$ Leiden and the G20210A prothrombin mutation. N Engl J Med 1999;341:801-6.

33. De Stefano V, Martinelli I, Mannucci P, et al. The risk of recurrent venous thromboembolism among heterozygous carriers of the G20210A prothrombin gene mutation. Br J Haematol 2001;113:630-5.

34. Baglin T, Luddington R, Brown K, Baglin C. Incidence of recurrent venous thromboembolism in relation to clinical and thrombophilic risk factors: prospective cohort study. Lancet 2003;362:523-6.

35. Simioni P. Risk for subsequent venous thromboembolic complications in carriers of the prothrombin or the factor $\mathrm{V}$ gene mutation with a first episode of deep-vein thrombosis. Blood 2000;96:3329-33.

36. Miles JS. G20210A mutation in the prothrombin gene and the risk of recurrent venous thromboembolism. J Am Coll Cardiol 2001;37:215-8.

37. Martinelli I, Bucciarelli P, Margaglione M, De Stefano V, Castaman G, Mannucci P. The risk of venous thromboembolism in family members with mutations in the genes of factor $\mathrm{V}$ or prothrombin or both. Br J Haematol 2000;111:1223-9.

38. Buller HR, Agnelli G, Hull RD, et al. Antithrombotic therapy for venous thromboembolic disease: the Seventh ACCP Conference on Antithrombotic and Thrombolytic Therapy. Chest 2004;126:401S. 